\title{
A Study on ECG Signal Classification Techniques
}

\author{
R. Kavitha \\ Assistant Professor \\ Dept of computer science \\ PSGR Krishnammal College for Women, \\ Coimbatore \\ India
}

\author{
T Christopher, Ph.D \\ Associate Professor \& Head \\ Dept of Computer Science \\ Govt Arts College, Udumalpet \\ India
}

\begin{abstract}
The abnormal condition of the electrical activity in the heart is using electrocardiogram shows a threat to human beings. It is a representative signal containing information about the condition of the heart. The P-QRS-T wave shape, size and their time intervals between its various peaks contain useful information about the nature of disease affecting the heart. This paper presents a technique to examine electrocardiogram (ECG) signal, by taking the features form the heart beats classification. ECG Signals are collected from MIT-BIH database. The heart rate is used as the base signal from which certain parameters are extracted and presented to the network for classification. This survey provides a comprehensive overview for the classification of heart rate.
\end{abstract}

\section{Keywords}

ECG Signal, MIT BIH Database, PQRST Wave

\section{INTRODUCTION}

The heart signals are taken from ECG, which is known as Electrocardiography. That the heart signals are picked by using electrodes in arms, leg, chest of our body. By using this signal heart disorder can be find out. Depend on the shape of the ECG waveform, find out the cardiac health. ECG signal readings and their analysis are carried out from signal processing. Today signal processing plays a major role in ECG signal analysis and interpretation. The aim of ECG signal processing is diverse and comprises the Improvement of measurement accuracy and reproducibility (when compared with manual measurements) and by taking out the information is not readily available from the signal through visual assessment.

In ECG signal noises are present in various situations, when taking records some type of instrumental noises are affected and also if the electrodes are not placed properly means noise will affect the signal. Various types of noises are present in the signal by Electrodes, Power interference, Instruments. So noise reduction in the ECG signal is most important aim of the signal processing.

Various methods are carried out by many researchers to find out the ECG characteristics [2]-[5]. One of the most important tools recently to remove the noise in the ECG signal by using wavelet transforms. This method also finds out the characteristic feature of the ECG signal wave form and obtains high accuracy. In wavelet transform various types are present that are dyadic, orthogonal, biorthogonal and continuous. In these dyadic wavelet transform provide better accuracy because of its good temporal localization property.

This paper gives the survey for ECG signal. In section II provide the review for Waveform detection. Section III provides Classification of Heart Rate. Comparisons of various methods are provided in section IV as in a tabulate form. Problems and direction are provided in Section V.

\section{WAVEFORM DETECTION}

In [6] the author finds out the QRS detection and intervals for RR by using Slope vector Waveform. In this methodology, to understand the required slope vector for ECG feature Extraction they used the variable stage differentiation. In this paper they got the better Signal to Noise ratio by using nonlinear amplification. $\mathrm{R}$ location is correctly find out by this type of technique and also fastly detected. Finally this paper capture QRS advanced duration, RR interval and Feature extraction fastly and gives better result. So as to induce QRS durations, the feature extraction rules are required.

Various number of algorithms are developed last 20 years because the essential importance of the QRS detection in the ECG analysis. The algorithms are used by filter bank, neural network, wavelet transform, and other methods are implemented [7]. Mostly linear and non- linear filters are used for the detection of the QRS [8]. It is one of the very simple methods to detect the QRS signal and also it is less computational power and is mostly suitable for in embedded real-time monitoring applications.

In [9] also they used the non-linear filters to detect the QRS complex. Less time have to be taken for this detection of QRS complex and also is one of the easy method. Even though these algorithms have some disadvantage to detect the QRS signal, that is some variation are happen in their frequency so it affects their performance. Results of these methods have high false positives and false negatives during the detection process, because the frequency band noise are larger than the frequency band of QRS complexes. In [10] cubic spline wavelets are used to detect the QRS waveform very accurately. From the result it produced highest accuracy reading in MIT-BIH arrhythmia database.

In [11] using filter to detect the QRS complex and their peaks. New method was implemented in [12, 13] upon filters, thresholding also used to detect the QRS complex, peak and $\mathrm{P}$ and $\mathrm{T}$ wave boundaries. Because of the low slope and magnitude of the $\mathrm{P}$ and $\mathrm{T}$ waves, as well as the presence of error such as noise, interference, and base line fluctuation, $\mathrm{P}$ and $\mathrm{T}$ wave delineation remains a difficult task. Furthermore, in addition to explanation, accurate estimation of the waveform itself may be important, e.g., for $\mathrm{T}$ wave alternans detection [14].

In [14] two type of methods are used they are post processing and preprocessing to detect the QRS and remove the noise in ECG signal. First preprocessing are taken place to detect the QRS beats by using threshold, four type of different threshold are used in this stage. Next post processing is take place to remove the noise in the ECG signal that is appearing in the Rwave. Some of them to detect the R- wave by using wavelet especially d3-d5 are implemented in [15] and d4 is used in [16] for the detection of QRS peak. 
In [17] algorithm used for the detection of QRS complexities. The recognition of QRS complexes forms the origin for more or less all automated ECG analysis algorithms. The presented algorithm utilizes a modified definition of slope, of ECG signal, as the feature for detection of QRS. A succession of transformations of the filtered and baseline drift corrected ECG signal is used for mining of a new modified slopefeature.

One of the method in threshold is adaptive quantized threshold are handled in [18] to detect the QRS complex. First filtering the input ECG signal by using transformation. Then in the signal to find out the correct QRS signal by using Multiple Quantized Threshold. This type of algorithm is one of the modifications in slope to detect the QRS complex; ECG signal is taken as a feature.

To detect the onset and offset of QRS complex, $\mathrm{P}$ and $\mathrm{T}$ wave in 12 lead ECG, simply said to be as delineation that is proposed in [19]. Moving average algorithm was proposed in this paper to remove the noise in the ECG signal. They collect the 125 original 12 lead ECG signal with 5000 samples is taken to detect the QRS complex. In this paper they used various window sizes to remove the noise in ECG signal. Finally they achieve noise free ECG signal with good accuracy. Another one method to detect the QRS complex by using Time Recursive prediction technique [20]. In this method they used to detect the QRS detection by using 3 lead and 1 lead.

In [20] QRS waveform is detection through Time Recursive prediction technique. In this algorithm they demonstrate the usefulness of time recursive prediction technique for event detection. They used 3 lead and 1 lead for QRS detection.

Digital band pass filter was proposed in [21] to minimize the various noises in the ECG signal and also it detect the locations of QRS complex have the maximum variation in the slopes. The method of "Pan and Tompkins" was adopted to detect the QRS complex $[14,10]$. To detect the $\mathrm{R}$ waveform, variations in slope and adaptive threshold are applied.

Coherent averaging of the respective signal part of all regular heartbeats generated a representative $\mathrm{P}$ wave template. Subsequently, all regular and abnormal heartbeats were compared to this $\mathrm{P}$-wave template using linear regression analysis [22].

In [23] Z80 assembly language was proposed to detect the real time QRS. To reduce the noise by using band pass filter. By using slope, amplitude and width information, proposed algorithm detect the QRS. Another one method to detect the QRS complex [24] by using Wavelet transforms. That the QRS can be differentiating from $\mathrm{P}$ and $\mathrm{T}$ waves by multiscale features of the Wavelet transform. In [25] $\mathrm{R}$ wave are detected using Wavelet transform. In this method the author used the Daubechies and Symmetric differentiator for detect the $\mathrm{R}$ wave. Another method to detect the QRS wave form was proposed in [26] by using Difference Operation Method. Compared to existing method, DOM algorithm in [26] was fast and efficient one and also it does not need any mathematical operation for detect the waveform. The author split the algorithm in two ways, first to find out the $\mathrm{R}$ wave by using difference equation. By using this $\mathrm{R}$ Peak to find out the $\mathrm{Q}$ and $\mathrm{S}$ wave is take place in the second stage.

\section{CLASSIFICATION OF HEART RATE}

In [27] classification of heart rate is based on the Neural Network and fuzzy. That the heart rate is classified by using Heart Rate Variability (HRV). Some features are extracted from this HRV and it is given as an input to the Neural Network and Fuzzy equivalence for classification. In [28] differentiate the ECG waveform into normal and abnormal by using features and it is given to the input for the classification. Here the classification was taken placed by (Linear Discriminant Analysis) LDA and Artificial Neural Networks (ANN). In this Multilayer Perception was carried out in ANN. In this paper the author describes that the MLP of ANN Network proves better result than the LDA classification. Again MLP neural network was carried in [5] to find out the normal and abnormal heart beat. Artificial neural network adaptive multiple preceptors are used to detect and classify the normal and abnormal heart beat [5]. In this paper the author take 12 different abnormal heart beat for classification and also another aim of this paper to remove the nonlinear background noise.

Another one algorithm proves less computationally [6] used to detect the QRS complex and interval of RR. In this paper the author proposed Slope Vector Waveform (SVW) algorithm. Two different type of operation are present for this algorithm that is Variable stage differentiation and non-linear amplification. To acquired the desired slope vector for feature extractions are handled by Variable stage differentiation and to get better signal-to-noise-ratio by using non-linear amplification. Even though in the presence of noise in the signal, this algorithm provides outstanding QRS detection.

Principal component analysis (PCA) and several types of neural network structures are used to detect and classify heart beat presented in [29]. Results of this work that different neural network structures in order to find the best neural network structure for the classification of specific types of arrhythmias have been compared. Neural network was implemented to detect the ischemic arrhythmia episodes in the ECG signal [30]. The author used the PCA for dimensionally reduction, and gets the effective training and the input to the network was reduced. The paper [30] proves the better result than existing one of ESC ST-T database.

In [31] used to detect the two types of ECG patterns by using different types of multilayer neural network as a classifier. To classify the ECG signal by using different algorithm [32], in this paper to reduce the dimensionally by using PCA. This paper combines the cluster algorithm of FCA with PCA neural network and proves better classification than PCA neural network, FCM neural Network, Wavelet neural network. Comparison between different structures for heart arrhythmia detection algorithms based on neural network, fuzzy cluster, wavelet transform and principal component analysis, was carried out by [32]. K-Nearest algorithm was implemented in [22] is used as a classification to find out QRS Wave form. In this paper, calculating the Cumulants from the R peak of ECG signal and the classification obtained high accuracy.

In [35] presented the classification performance of an automatic classifier of the electrocardiogram (ECG) for the detection abnormal beats with new concept of feature extraction stage. Depend on the ECG morphology and RR intervals features sets are produced. Configuration adopted a Kohonen self organizing maps (SOM) for analysis of signal features and clustering. Classifier was developed with SOM and learning vector quantization (LVQ) algorithms using the data from the records recommended by ANSI/AAMI EC57 standard. Their paper compares two strategies for classification of annotated QRS complexes: based on original ECG morphology features and proposed new approach based on preprocessed ECG morphology features. The 
mathematical morphology filtering was used for the preprocessing of ECG signal. The performance of the algorithm was evaluated on the MIT-BIH Arrhythmia Database following the AAMI recommendations. By this method the results of recognition beats either as normal or arrhythmias was improved.

In [36] using Wavelet transformation and neural networks features are extracted from ECG signal. First features are extracted from wavelet decomposition and given the features as the input for the classification. Mean, Median, Maximum, Minimum, Standard Deviation, Range, Variance and Mean Absolute Deviation these are the features given to the Classification is done by neural network. With the help of these features Artificial Neural Network trained the various diseases in ECG signal and got the accuracy of $92 \%$.

Table 1. Comparison of Various Methods

\begin{tabular}{|c|c|c|}
\hline SNO & Description & Methods \\
\hline 1. & $\begin{array}{l}\text { Removal of Power Line Interference and other } \\
\text { Single Frequency Tones from Signals [37]. }\end{array}$ & $\begin{array}{l}\text { Number of methods has developed to remove the noise } \\
\text { in the signal. Several noises are acquired in the signal. } \\
\text { Baseline drift, Power line interferences, contact } \\
\text { electrodes these are some type of noises present in the } \\
\text { signal. There are number of techniques have been } \\
\text { developed to remove the unwanted noises in the } \\
\text { medical monitoring equipments especially Power line } \\
\text { interference. Several bioelectric signals are attacked by } \\
\text { power line interference (50/60 Hz) noise. It presents the } \\
\text { removal of power line interference and other single } \\
\text { frequency tones from ECG signal using the advanced } \\
\text { adaptive filtering Technique with LMS (least mean } \\
\text { square) algorithm }\end{array}$ \\
\hline 2 & $\begin{array}{l}\text { Genetic algorithm and wavelet hybrid scheme for } \\
\text { ECG signal denoising, [38] }\end{array}$ & $\begin{array}{l}\text { Introduce an effective hybrid scheme of genetic } \\
\text { algorithm and wavelet transform are used to remove } \\
\text { noise in the ECG signal }\end{array}$ \\
\hline 3. & $\begin{array}{l}\text { Processing ECG Signal With Kaiser Window- } \\
\text { Based FIR Digital Filter [39]. }\end{array}$ & $\begin{array}{l}\text { Designing FIR digital filters with Kaiser Window to } \\
\text { remove the interferences or the artifacts in the ECG } \\
\text { signal reduces noises. For this three type of filters are } \\
\text { considered low pass, high pass and notch filter to filter } \\
\text { the raw noisy ECG signal after this the three filters are } \\
\text { cascaded }\end{array}$ \\
\hline 4 & Optimized Noise Canceller for ECG Signals [40]. & $\begin{array}{l}\text { Various interferences are distorting the ECG signal in } \\
\text { the time of acquisition time. These types of noises are } \\
\text { reduced with the help of adaptive filters. Traditional } \\
\text { optimization techniques have been very popular } \\
\text { because of their advantages. One of the traditional } \\
\text { optimization techniques is Least Mean Square (LMS) } \\
\text { which is gradient based. Type of this method converges } \\
\text { very easy. }\end{array}$ \\
\hline 5 & $\begin{array}{l}\text { Arrhythmia ECG Noise Reduction by Ensemble } \\
\text { Empirical Mode Decomposition [41]. }\end{array}$ & $\begin{array}{l}\text { To remove some of the artifacts in raw ECG signal by } \\
\text { using one of the noise filtering of Ensemble Empirical } \\
\text { Mode Decomposition (EEMD). For this IIR filter, } \\
\text { Wiener filter, empirical mode decomposition (EMD) } \\
\text { and EEMD were used to compare filtering } \\
\text { Performance. }\end{array}$ \\
\hline 6 & $\begin{array}{l}\text { Digital Filtration of ECG Signals for Removal of } \\
\text { Baseline Drift [42]. }\end{array}$ & $\begin{array}{l}\text { Processing of ECG signal analysis Baseline wander is } \\
\text { one of the major steps. The detection of ST segment in } \\
\text { the baseline wander is very difficult. So to remove the } \\
\text { baseline wander by using digital filters. }\end{array}$ \\
\hline
\end{tabular}




\begin{tabular}{|c|c|c|}
\hline 7 & $\begin{array}{l}\text { Comparative study of FIR and IIR filters for the } \\
\text { removal of Baseline noises from ECG signal } \\
\text { [43]. }\end{array}$ & $\begin{array}{l}\text { Filter bank such are IIR and FIR filter also used to } \\
\text { eliminate the noise in the ECG signal. Compared to Fir } \\
\text { filter IIR } 2 \text { is very easy to implement this algorithm to } \\
\text { remove the noise and also it is less computational } \\
\text { power. For baseline drift elimination IIR filter is a } \\
\text { better one to remove this type of filter. }\end{array}$ \\
\hline 8 & $\begin{array}{l}\text { On Baseline Drift Suppressing in ECG Recording } \\
\text { [44]. }\end{array}$ & $\begin{array}{l}\text { Real-time bidirectional baseline drift suppression filter } \\
\text { for multiple lead ECG. This type of filter have minimal } \\
\text { delay, minimal non-linear phase Shift, minimal } \\
\text { calculation power and maximal signal-to-noise ratio } \\
\text { and minimal ECG-signal }\end{array}$ \\
\hline 9 & $\begin{array}{l}\text { The Combined Effect of Median and FIR Filter in } \\
\text { Pre-processing of ECG Signal using Matlab [45]. }\end{array}$ & $\begin{array}{l}\text { Combined effect of Median and FIR filter for the } \\
\text { preprocessing of an ECG signal is used to remove the } \\
\text { noise from the ECG signal. } \\
\text { Not only removes the baseline drift also preserves } \\
\text { edges while removing noise. }\end{array}$ \\
\hline 10 & $\begin{array}{l}\text { Adaptive Baseline Wander Removal in the ECG: } \\
\text { Comparative Analysis With Cubic Spline } \\
\text { Technique [46]. }\end{array}$ & $\begin{array}{l}\text { To remove the baseline drift by using cascade adaptive } \\
\text { filter. These cascade filters have two types of stages. } \\
\text { The initial stage was as adaptive notch filter at zero } \\
\text { frequency. Then the next stage used to detect the QRS } \\
\text { by using an adaptive impulse correlated filter. } \\
\text { This type of method removes base line drift in real time } \\
\text { without calculating the electric levels. }\end{array}$ \\
\hline 11 & $\begin{array}{l}\text { QRS Complex Detection of Ecg Signal Using } \\
\text { Wavelet Transform [47]. }\end{array}$ & $\begin{array}{l}\text { By subtracting the filtered signal from the original } \\
\text { signal, a signal with baseline drift elimination can be } \\
\text { obtained. That the filtered signals are obtained from } \\
\text { median filter. After remove the baseline drift applying } \\
\text { digital filters and wavelet transform for to remove some } \\
\text { of the noises in the ECG signal. }\end{array}$ \\
\hline 12 & Atrial fibrillation detection using P-Wave [48] & $\begin{array}{l}\text { By using the Mean, Minimum, Maximum, Standard } \\
\text { Deviation of the amplitude, area, width, time distance } \\
\text { of the P-wave, Atrial fibrillation detection are find. }\end{array}$ \\
\hline 13 & $\begin{array}{l}\text { Detection of Atrial fibrillation using } \\
\text { complex. [49] }\end{array}$ & $\begin{array}{l}\text { Area of the QRS complex are used to detect the Atrial } \\
\text { Fibrillation, these are find out from the amplitude of the } \\
\mathrm{R} \text { peak and the total time duration for the QRS complex } \\
\text { using trapezoidal method. }\end{array}$ \\
\hline
\end{tabular}

\section{PROBLEMS AND DIRECTIONS}

Some of the Existing methods have limitations in their heart rate ECG classification. Such a limitations are accuracy, removal of noises and detection of peaks in ECG signal and Heart rate. In [50] they produce accuracy of $85 \%$ using artificial neural network, which is very low. In this paper the author used two type of classification for classify the features. To overcome this only one classifier are used to classify the features and also give the better accuracy of above $95 \%$ by using RVM. Some of the developing algorithm didn't provide the fast extraction features from the ECG signal. To overcome this QRS complex methods are used to extract the fast feature extraction from the ECG signal. In [45] they remove the base line drift by using combination of Median filter and FIR filter, it is extendable process. To overcome this only one type of filter is used to remove the base line drifts and also some of the edges in the ECG signal. 


\section{CONCLUSION}

In this paper, a novel ECG beat classification algorithms are proposed and the signals are taken from the MIT/BIH data base. Provide a brief survey on ECG classification. The examination of the ECG has been comprehensively used for diagnosing many cardiac diseases. Various techniques and transformations have been proposed earlier in literature for ECG signal. This proposed paper provides an over view of Noise removal, Waveform detection and classification of the Heart rate. This paper also revealed a comparative table evaluating the performance of different algorithms that were proposed earlier for ECG signal. And also provide the problem of the existing work and gives the direction for this also. We noticed that the most of the work for the removal of noise they used combination of filters. Most of the uses QRS complex for heart rate classification. The future work which can be done is to make an adaptive preprocessing and classifier system for the ECG classification.

\section{REFERENCES}

[1] Leif Sornmo, Pablo Laguna., Electrocardiogram (ECG) Signal Processing".

[2] Pan, J. and Tompkins, W. J. 1985 A real-time QRS detection algorithm, IEEE Trans. Biomed. Eng., vol. 32, pp. 230-236.

[3] Afonso, V.X., Tompkins, W.J., Nguyen, T.Q and S. Luo. 1999. ECG beat detection using filter banks, IEEE Trans. Biomed. Eng., vol. 46, pp. 192-202.

[4] Bahoura, M., Hassani, M. and Hubin, M. 1997. DSP implementation of wavelet transform for real time ECG wave forms detection and heart rate analysis, Comput. Methods Programs Biomed, vol. 52, no. 1, pp. 35-44.

[5] Hu, Y.H., Tompkins, W.J., Urrusti, J.L and Afonso, V.X. 1993. Applications of artificial neural networks for ECG signal detection and classification, J .Electro cardiology, vol. 26 (Suppl.), pp. 66-73.

[6] Xiaomin, Xu. and Ying, Liu. 2004. ECG QRS Complex Detection Using Slope Vector Waveform (SVW) Algorithm, Proceedings of the26th Annual International Conference of the IEEE EMBS, pp. 3597-3600.

[7] Okada, M. 1979. A digital filter for the QRS complex detection, IEEE Trans. Biomed. Eng., Vol. BME-26, pp. 702-703.

[8] Afonso, V. X. and Tompkins, W. J. 1999. ECG Beat Detection Using Filter Banks, IEEE Trans. Biomed. Eng., Vol. 46, No. 2, pp. 192-202.

[9] Kohler, BU., Henning, C and Orglmeister, R. 2002. The principles of software QRS detection, IEEE Eng. Med. Biol. Mag., vol. 21, no. 1, pp. 42-57.

[10] Dinh, HAN., Kumar, DK., Pah, ND and Burton, P. 2001.Wavelets for QRS detection, in Proc. 23rd IEEE EMBS Int. Conf, pp. 1883- 1887.

[11] Laguna, P., Jane, R and Caminal, P. 1994. Automatic detection of wave 'boundaries in multi lead ECG signals: Validation with the CSE database, Comput. Biomed. Res., vol. 27, no. 1, pp. 45-60.

[12] Martinez, J.P., Almeida, R., Olmos, S., Rocha, AP and Laguna, P. 2004. A wavelet-based ECG delineator: Evaluation on standard databases, IEEE Trans. Biomed. Eng., vol. 51, no. 4, pp. 570-581.
[13] Chouhan, V.S and Mehta, S.S. 2008. Threshold-based detection of $\mathrm{P}$ and $\mathrm{T}$-wave in ECG using new feature signal, Int. J. Comp. Science Net. Security, vol. 8, no. 2, pp. $144-152$.

[14] Martinez, J.P and Olmos, S. 2004. Methodological principles of $\mathrm{T}$ wave alternans analysis: A unified framework, IEEE Trans. Biomed. Eng., vol. 52, no. 4 pp. 599-613.

[15] Cuiwei Li., Chongxun Zheng and Changfeng Tai. 1995 Detection of ECG Characteristic points using Wavelet Transforms, IEEE Trans. Biomed. Eng, Vol. 42, No. 1.

[16] Mahmoodabadi, S.Z., Ahmadian, A., Abolhasani, M.D., Eslami, $M$ and Bidgoli, J.H. 2005. ECG Feature Extraction Based on Multiresolution Wavelet Transform, Proceedings of the 2005 IEEE, Engineering in Medicine and Biology 27th Annual Conference Shanghai, China, September 1-4.

[17] Saxena, SC., Kumar, V and Hande, ST. 2002. QRS Detection using New Wavelets, Journal of Medical Engineering \& Technology, Volume 26, November1, pages 7-15.

[18] Chouhan, V.S and Mehta, S.S. 2008. Detection QRS Complex in 12 lead ECG using Adaptive Quantized Threshold, International Journal of Computer Science and Network Security, Vol 8 No.1.

[19] Chouhan, V.S., Mehta, SS and Ligayat, N.S. 2008 Delineation QRS Complex, $\mathrm{P}$ and $\mathrm{T}$ wave in $12 \mathrm{Lead}$ ECG'IJCSNS Vol. 8 No.4.

[20] Kyrkos, A., Giakoumakis, E.A and Carayannis, G. 1998 QRS Detection through Time Recursive Prediction Technique, Signal Processing 15(1988) 429-436.

[21] Pan, J and Tompkins, W. 1985. A Real Time QRS Detection Algorithm, IEEE Transaction sons on Biomedical Engineering, vol. 32, no. 3, pp. 230- 236.

[22] Schreicr, G., Kastner, P. and Marko, W. 2001. An Automatic ECG Processing Algorithm to Identify Patients Prone to Paroxysmal Atrial Fibrillation, IEEE Computers in Cardlology, vol. 28, pp. 133- 135.

[23] Jaipupan and Tompkins, W. 1985. A Real-Time QRS Detection Algorithm, IEEE Transaction on Biomedical Engineering, Vol, Bme-32, No3.

[24] Li, C.W., Zheng, C.X and Tai, C.F. 1995. Detection of ECG characteristic points using wavelet transforms, IEEE Trans. Biomed. Eng. 42 (1) (1995), pp 21-28, (1995)

[25] Awadhesh Pachauri and Manabendra Bhuyan., Robust Detection of R-Wave Using Wavelet Technique, World Academy of science, Engineering and technology 56(2009)

[26] Yeh, Y.C and Wang, W.J. 2008. QRS Complexes detection for ECG signal: The Difference Operation Method", Elsevier Journal, Computer Methods and Programs in Biomedicine, 245-254, (2008)

[27] Rajendra Acharya, U., Subbanna Bhat, P., Iyengar, S.S 2003. Ashok Rao and Sumeet Dua., Classification of heart rate data using artificial neural network and fuzzy equivalence relation", Pattern Recognition 36 (2003) 61 -68 . 
[28] Alexakis, C., Nyongesa, HO., Saatchi, R., Harris, ND., Davies, C., Emery, C., Ireland, RH and Heller SR. 2003. Feature Extraction and Classification of Electrocardiogram (ECG) Signals Related to Hypoglycemia", Conference on computers in Cardiology, pp. 537-540, IEEE.

[29] Silipo, R and Marchesi, C. 1998. Artificial neural networks for automatic ECG analysis, Signal Processing 1998. 46; 1417-1425.

[30] Papaloukas, C and Fotiadis, D.I. 2002. An ischemia detection method based on artificial neural network, Artificial Intelligence in Medicine 2002; 24: 167-178.

[31] Foo, SY and Stuart, G. 2002. Neural network-based ECG pattern recognition, Engineering Applications of Artificial Intelligence 2002; 15: 253-260.

[32] Ceylan, R and Ozbay, Y. 2007. Comparison of FCM, PCA and WT techniques for classification ECG arrhythmias using artificial neural network, Expert Systems with Applications, 286-295.

[33] Kutlu, Y and Kuntalp, D 2008. Arrhythmia classification using higher order statistics, IEEE Signal Processing, Communication and Applications Conference 2008; IEEE Press: $1-4$.

[34] Cvikl, M and Zemva, A. 2010. FPGA-oriented HW/SW implementation of ECG beat detection and classification algorithm”, Digital Signal Processing 2010; 20: 238-248, (2010)

[35] Tadejko, P and Rakowski, W. 2007. Mathematical Morphology Based ECG Feature Extraction for the Purpose of Heartbeat Classification, 6th International Conference on Computer Information Systems and Industrial Management Applications, CISIM '07, pp. 322-327

[36] Tayel, M.B and El-Bouridy, M.E. 2006. ECG Images Classification Using Feature Extraction Based On Wavelet Transformation And Neural Network, ICGST, International Conference on AIML.

[37] Tareen, S.G. 2008. Removal of Power Line Interference and other Single Frequency Tones from Signals, M.Sc, Computer Science and Electronics, Mälardalen University, sweedan.

[38] El-Dahshan, ESA. 2010. Genetic algorithm and wavelet hybrid scheme for ECG signal denoising, Telecommunication Systems, vol. 46, pp. 209-215.

[39] Mbachu, C.B., Onoh, G.N., Idigo V.E., Ifeagwu E.N and Nnebe S.U. 2011. Processing ECG Signal with Kaiser Window- based FIR Digital Filters" International Journal of Engineering Science and Technology, Vol. 3 No. 8, 6775-6783.

[40] Dutta, SSDM. 2011. Optimized Noise Canceller for ECG Signals, International Conference on Intelligent Systems and Data Processing (ICISD).

[41] Chang, K.M. 2010. Arrhythmia ECG Noise Reduction by Ensemble Empirical Mode Decomposition, Sensors, 2010, doi:10.3390/s100606063

[42] Kaur, M., Singh, B., Ubhi, JS and Seema Rani. 2011 Digital Filteration of ECG Signals for Removal of Baseline Drift", 2011 International Conference on Telecommunication Technology and Applications Proc .of CSIT vol.5 pg no.105-109.

[43] Seema Rani, Amarpreet kaur and Ubhi, J.S. 2011. Comparative study of FIR and IIR filters for the removal of Baseline noises from ECG signal, International Journal of Computer Science and Information Technologies Vol 2 (3).

[44] Harting, L.P., Fedotov, N.M and Slump, C.S. 2004. On Baseline Drift Suppressing in ECG Recording” 2004 IEEE Benelux Signal Processing Symposium.

[45] Karnewar, J.S and Sarode, M.V. 2013. The Combined Effect of Median and FIR Filter in Pre-processing of ECG Signal using Matlab, International Journal of Computer Applications (0975 - 8887), National Level Technical Conference "X-PLORE 13.

[46] Jane, R., Laguna, P., Thakor, N.V and Caminal, P. 1992. Adaptive Baseline Wander Removal in the ECG Comparative Analysis with Cubic Spline Technique' IEEE proceeding Computers in Cardiology, pp.143- 146.

[47] Priyanka Mehta and Monika Kumari. 2012. QRS Complex Detection of ECG Signal Using Wavelet Transform", International Journal of Applied Engineering Research, Vol.7 No.11.

[48] Ros, E., Mota, S., Fernandez, F., Toro, F and Bernier, J. 2004. ECG characterization of paroxysmal Atria fibrillation: Parameter extraction and automatic diagnosis algorithm, Computers in Biology and Medicine, vol. 34, no. 8 , pp. $679-696$.

[49] Hickey, B., Heneghan, C and Chazal, P.D. 2004. Nonepisode-dependent assessment of paroxysmal atrial fibrillation through measurement of RR interval dynamics and atrial premature contractions, Annals of Biomedical Engineering, vol. 32, no. 5, pp. $677-687$.

[50] Rajendra Acharya, U., Subbanna Bhat, P., Iyengarc, SS., Ashok Rao, Sumeet Dua. 2002. Classification of heart rate data using artificial neural network and fuzzy equivalence relation", Pattern Recognition Society. 